Submitted to European Polymer Journal as Regular Article 24/11/2017 Special Issue "Polymer Crystallization: From Classical Systems to Functional Materials and Biopolymers"

Revised xx/01/2018

\title{
Smectic liquid crystal Schlieren texture in rapidly cooled poly(butylene naphthalate)
}

Qian Ding ${ }^{1,2}$, Dieter Jehnichen ${ }^{3}$, Michael Göbel ${ }^{3}$, Michelina Soccio ${ }^{4}$, Nadia Lotti ${ }^{4}$, Dario Cavallo ${ }^{5 *}$, René Androsch ${ }^{1 *}$

1 Interdisciplinary Center for Transfer-oriented Research in Natural Sciences (IWE TFN), Martin Luther University Halle-Wittenberg, 06099 Halle/Saale, Germany

2 School of Packing Design and Art, Hunan University of Technology, 88 Taishan West Road, Zhuzhou 412007, P.R. China

3 Leibniz-Institut für Polymerforschung Dresden e.V., Hohe Str. 6, 01069 Dresden, Germany

4 Civil, Chemical, Environmental and Materials Engineering Department, University of Bologna, Via Terracini 28, 40131, Bologna, Italy

5 Department of Chemistry and Industrial Chemistry, University of Genova, Via Dodecaneso 31, 16146 Genova, Italy

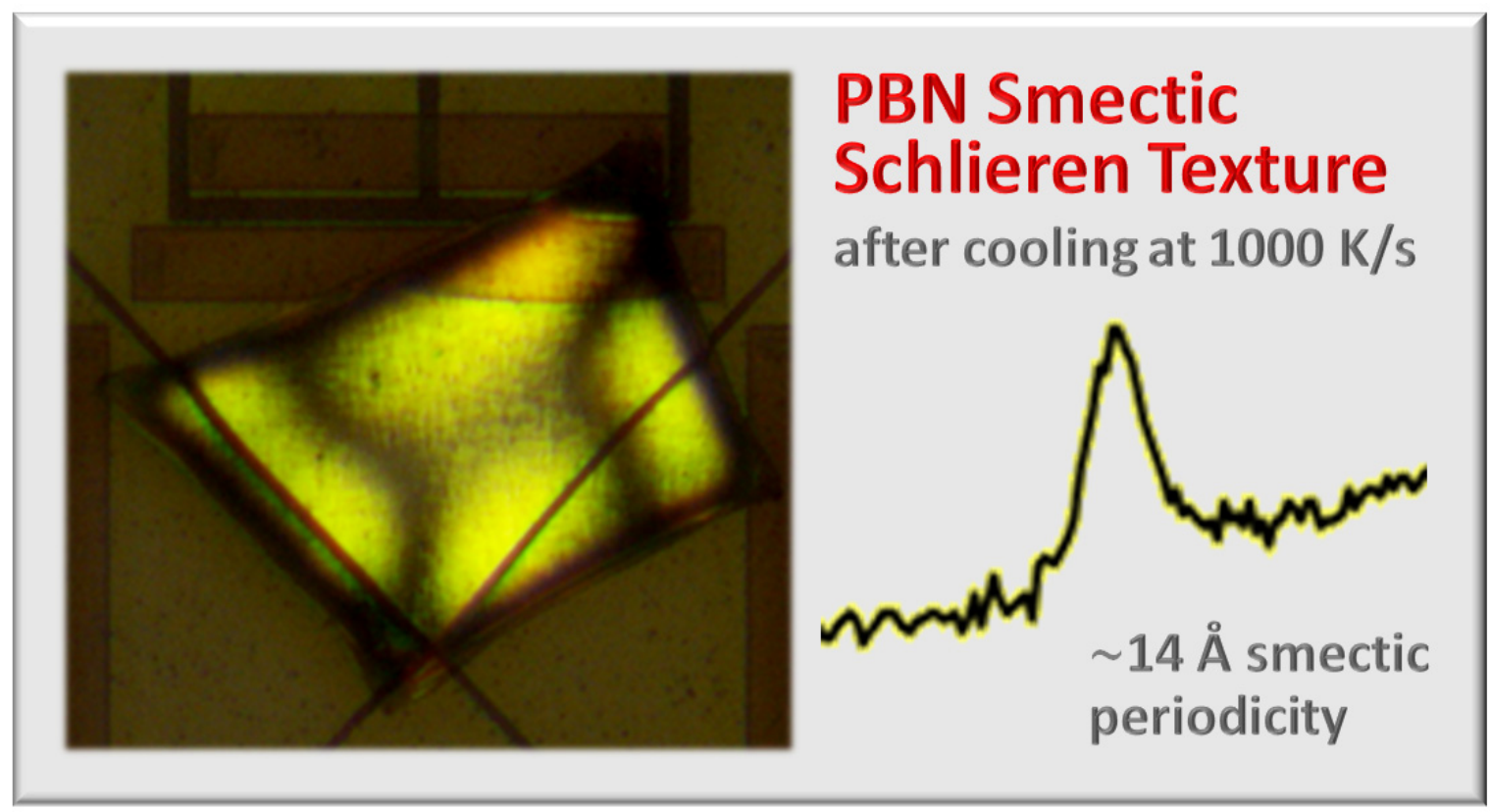

\footnotetext{
* Corresponding Authors: dario.cavallo@unige.it; rene.androsch@,iw.uni-halle.de 


\title{
Highlights
}

Melt-crystallization of PBN was analyzed as a function of the crystallization conditions.

Crystallization above $160{ }^{\circ} \mathrm{C}$ leads to formation of crystals and spherulites directly from the melt.

Crystallization below $160^{\circ} \mathrm{C}$ proceeds via intermediate formation of a smectic liquid crystalline (LC) phase.

Cooling faster than $200-500 \mathrm{~K} / \mathrm{s}$ suppresses crystal formation from the LC phase.

The smectic LC phase exhibits a distinct Schlieren texture.

\begin{abstract}
The morphology of partially crystalline/ordered poly(butylene naphthalate) (PBN) forming on cooling the melt has been analyzed by polarized-light optical microscopy (POM) and microfocus-beam X-ray diffraction (XRD). Crystallization at rather low supercooling of the melt, at temperatures higher than about $200^{\circ} \mathrm{C}$, leads to slow and irregular spherulitic growth of $\beta$ '-crystals, with spherulites not showing a distinct Maltese cross in POM. At temperatures between approximately 200 and $160{ }^{\circ} \mathrm{C}$, the melt partially converts directly to $\alpha$-crystals, and the obtained spherulitic superstructure reveals an increasing nuclei density with decreasing crystallization temperature. At even lower temperature, a liquid crystalline (LC) phase develops. This mesophase may subsequently convert to $\alpha$-crystals according to Ostwald's rule of stages. The transition of the LC-phase into $\alpha$-crystals is suppressed at temperatures lower than about $120^{\circ} \mathrm{C}$ or on cooling faster than about $200-500 \mathrm{~K} / \mathrm{s}$. X-ray analysis of PBN liquid crystals formed at well-defined cooling conditions in a fast scanning chip calorimeter revealed smectic periodicity while there is simultaneously observed a distinct Schlieren texture in POM.
\end{abstract}

Keywords: Poly(butylene naphthalate); Liquid crystal; Crystallization; Fast scanning chip calorimetry; Morphology 


\section{Introduction}

Poly(butylene naphthalate) (PBN) is an engineering high-performance polymer which contains a rigid naphthalene ring and flexible methylene sequence in its repeating unit. It is characterized by superior wear and sliding characteristics, hydrolysis and chemical resistance, excellent gas barrier properties, as well as high temperature stability [1,2]. The properties and therefore also potential applications of this polyester are expected to depend on the semicrystalline morphology which typically forms on cooling during meltprocessing. However, the effect of the solidification conditions, encompassing the formation of different ordered phases, on the final morphology at ambient temperature, is still not clearly established. Partial ordering of PBN can only be avoided on cooling the melt at rates higher than $6000 \mathrm{~K} / \mathrm{s}$ to below the glass transition temperature $\left(T_{\mathrm{g}}\right)$, as recently shown in a study of the vitrification and crystal-nucleation behaviors employing fast scanning chip calorimetry (FSC) [3]. Slower cooling causes the formation of crystals or of a mesophase depending on the specific cooling rate/supercooling of the melt before the phase transition.

In detail, it has been suggested that slow cooling of the melt at rates slower than $10 \mathrm{~K} / \mathrm{min}$ $(0.167 \mathrm{~K} / \mathrm{s})$, or crystallization at temperatures higher than about $200{ }^{\circ} \mathrm{C}$, leads to the development of $\beta$ '-crystals [4-6]. The $\beta$ '-phase exhibits a triclinic unit cell $\left(\mathrm{a}_{0}=0.455 \mathrm{~nm}\right.$, $\mathrm{b}_{0}=0.643 \mathrm{~nm}, \mathrm{c}_{0}=1.531 \mathrm{~nm}, \alpha=110.1^{\circ}, \beta=126.9^{\circ}, \gamma=100.6^{\circ}$ [7]) in which the butylene units adopt a nearly all-trans chain conformation [8-10], and an equilibrium melting temperature of $281^{\circ} \mathrm{C}$ [11]. Morphological information about the $\beta$ '-crystal polymorph is rare. We are aware of a single study in which it was shown that $\beta$ '-crystals "grow radially and almost individually from the center" of dendritic spherulites [6], with such morphology attributed to the very slow crystal growth rate. Formation of $\beta$ '-crystals is increasingly replaced by formation of triclinic $\alpha$-crystals $\left(a_{0}=0.487 \mathrm{~nm}, b_{0}=0.622 \mathrm{~nm}\right.$, $\mathrm{c}_{0}=1.436 \mathrm{~nm}, \alpha=110.78^{\circ}, \beta=121.10^{\circ}, \gamma=97.93^{\circ}$ [7]) on increasing the cooling rate to values higher than $0.1 \mathrm{~K} / \mathrm{min}(0.017 \mathrm{~K} / \mathrm{s})$, or decreasing the crystallization temperature to below $230{ }^{\circ} \mathrm{C}$. If the cooling rate exceeds $10 \mathrm{~K} / \mathrm{min}(0.167 \mathrm{~K} / \mathrm{s})$, or if the crystallization temperature is lower than about $200{ }^{\circ} \mathrm{C}$, then only $\alpha$-crystals are forming. The equilibrium melting temperature of the $\alpha$-phase is $261^{\circ} \mathrm{C}$, that is, about $20 \mathrm{~K}$ lower than that of $\beta$ 'crystals, as was ascribed to the lower packing density [11]. Note that further reports about equilibrium melting temperatures of PBN crystals of $276^{\circ} \mathrm{C}$ [6] and $294{ }^{\circ} \mathrm{C}$ [12] are available, however, without any assignment to a specific crystal polymorph. The main 
difference of the crystal structure of the $\alpha$-crystals compared to the $\beta$ '-phase is the conformation of the butylene unit, leading to a shorter fiber identity period (FIP). Growth of $\alpha$-crystals is reported being connected with the formation of typical spherulites [6]. If the cooling rate is increased further, as in an early experiment has been achieved by quenching of a $150 \mu \mathrm{m}$ thin film into ice-water [13], then formation of $\alpha$-crystals is suppressed and replaced by the formation of a mesophase. The mesophase has been described being nearly transparent, and possessing a liquid-crystalline (LC) structure with the molecules adopting the same local chain conformation as in $\alpha$-crystals. Analysis of the X-ray fiber pattern obtained for a stretched sample led to the conclusion that the PBN mesophase is a smectic-A liquid crystal with a smectic periodicity (layer distance) of 1.43 $\mathrm{nm}$. Importantly, the smectic LC phase has been suggested to exist only below the glass transition temperature of the system, possessing a frozen liquid crystalline structure. As such, the mesophase is more precisely classified as an LC glass [14, 15]; heating the LC glass to above its $T_{\mathrm{g}}\left(65^{\circ} \mathrm{C}\right)$ at a rate of $4 \mathrm{~K} / \mathrm{min}$ caused transformation into $\alpha$-crystals at slightly higher temperature. Later on, the conditions of mesophase formation were further evaluated by FSC and fast X-ray diffraction experiments [16]. It was found that on cooling the melt at rates between 2400 and $24000 \mathrm{~K} / \mathrm{min}$ (40 and $400 \mathrm{~K} / \mathrm{s}$ ) the mesophase forms as an intermediate transient stage within the path of transformation of the melt into $\alpha$-crystals, following Ostwald's rule of stages [17]. This crystallization pathway in which the isotropic melt first transforms into an LC-phase, which undergoes a monotropic transition into a more ordered crystal phase, is not uncommon in aromatic polyesters where the stiff aryl rings act as mesogenic units [18-23]. However, in case of PBN the transition of the LCphase into crystals is finally suppressed if the cooling rate exceeds $24000 \mathrm{~K} / \mathrm{min}(400 \mathrm{~K} / \mathrm{s})$, leading to the above described formation of an LC glass.

For polymers forming a monotropic nematic structure, polarized-light optical microscopy $(\mathrm{POM})$ revealed a fine-grained structure of the LC-phase at the micrometer scale [19-21], modeled as isolated nematic domains embedded in the isotropic phase [22]. Similarly, for a monotropic smectic LC-phase such fine-grained structure was also detected [23]. In the subsequently occurring crystallization process of the nematic and smectic phase, these liquid crystalline structures serve as precursors/nuclei, distinctly increasing the crystallization rate while preserving the initially formed grainy morphology [19-23]. For PBN, however, morphological information about the smectic mesophase is not available yet, which is therefore provided in the present study. 


\section{Experimental}

PBN was obtained from Teijin Shoji Europe GmbH in form of additive-free pellets. The intrinsic viscosity of the polymer is $0.92 \mathrm{dL} / \mathrm{g}$ and was measured at $30^{\circ} \mathrm{C}$ using a mixture of phenol and 1,1,2,2-tetrachloroethane $60: 40 \mathrm{w} / \mathrm{w}$.

Differential scanning calorimetry (DSC) was used to obtain crystallization temperatures on cooling at rates lower than about $1 \mathrm{~K} / \mathrm{s}$. We employed a Mettler-Toledo DSC 1, operated in conjunction with a Huber TC 100 intracooler, and with the furnace purged using nitrogen gas at a flow rate of $60 \mathrm{~mL} / \mathrm{min}$. A sample with a mass of about $5 \mathrm{mg}$ was encapsulated in a $20 \mu \mathrm{L}$ aluminum pan and heated to $280{ }^{\circ} \mathrm{C}$ to obtain a relaxed melt within three minutes holding time, before cooling at different rates. To assure absence of degradation during repeated heating and cooling, reproducibility of crystallization temperatures was checked. Furthermore, to exclude systematic instrumental errors, a Mettler-Toledo DSC 820 was used to confirm the obtained results.

Analysis of non-isothermal crystallization on cooling at rates between 1 and $5000 \mathrm{~K} / \mathrm{s}$ was done using a Mettler-Toledo FSC connected to a Huber intracooler TC 100, and using dry nitrogen gas to purge the sample at a flow rate of $40 \mathrm{~mL} / \mathrm{min}$. Sensors were conditioned and temperature-corrected before loading with specimens of different mass, using Wacker silicon oil AK 60,000 as contact medium towards the sensor membrane. Samples were prepared from the as-received pellets using a microtome to obtain thin sections with a thickness of less than $20 \mu \mathrm{m}$ which then were further reduced in their lateral width to about 50-100 $\mu \mathrm{m}$ using a scalpel and a stereomicroscope.

POM was employed to obtain morphological data at the micrometer length scale of PBN of different thermal history. Thin sections with a thickness of about $20 \mu \mathrm{m}$ were prepared using a Slee microtome, placed between Plano covers slips and melted at $290{ }^{\circ} \mathrm{C}$ on a hot stage. Then the glass-polymer-glass sandwich was either quickly transferred to a second hot stage to allow isothermal crystallization at pre-adjusted target temperatures, or quenched in ice water to achieve fast cooling. The morphology of the samples was then evaluated between crossed polarizers in a Leica DMRX optical microscope in transmission mode, using a Motic CCD camera for imaging.

For the precise analysis of the effect of the rate of cooling the melt of PBN on the formation of the various possible polymorphs, X-ray diffraction (XRD) patterns of FSC samples with a well-defined cooling history have been collected using a SAXSLAB ApS Ganesha $300 \mathrm{XL}+$ system (Denmark). The samples remained attached to the membrane of 
the FSC sensor for easy handling and were investigated in transmission mode, using monochromatic $\mathrm{Cu} \mathrm{K} \alpha$ radiation and a microfocus $\mathrm{X}$-ray beam with a cross-section of $400 \times 400 \mu \mathrm{m}^{2}$. For registration of the scattered X-rays, a Pilatus 300K 2D-detector was employed using an exposure time of $30 \mathrm{~min}$. Preferred crystal orientation was not detected, allowing azimuthal averaging of the $2 \mathrm{D}$ frames to get XRD curves without loss of information.

The morphology of FSC samples subjected to specific cooling pathways, finally, was evaluated by POM operated in reflection mode, using a Kern OPN 184 microscope equipped with a Leica imaging system.

\section{Results and discussion}

Figure 1 shows the dependence of the crystallization peak temperature on the cooling rate, together with information about the specific phases developing. Coloring of symbols and lines is used to emphasize formation of $\beta$ '- and $\alpha$-crystals from the isotropic melt (red), of $\alpha$-crystals from the LC-phase (blue), and of the LC-phase from the melt (gray/black). Cooling at rates lower than about $0.01 \mathrm{~K} / \mathrm{s}$ causes crystallization at temperatures higher than about $220^{\circ} \mathrm{C}$ and presumably, according to the literature [4-6], to the formation of $\beta$ 'crystals from the isotropic melt. With increasing cooling rate the crystallization temperature decreases, and formation of $\beta$ '-crystals should progressively be replaced by $\alpha$ crystals. For the specific PBN grade investigated the crystallization process at slow cooling occurs in a rather wide temperature range, as is indicated by the largely different onset (dotted red line) and peak temperatures (red symbols, solid line). Cooling at a rate of about $1 \mathrm{~K} / \mathrm{s}$ leads to a slow crystallization process beginning at a temperature slightly higher than $200{ }^{\circ} \mathrm{C}$ which then is superimposed by a fast event at about $160{ }^{\circ} \mathrm{C}$ (see red-blue colored vertical arrow). At present, it may be assumed that $\alpha$-crystal formation begins at around $200{ }^{\circ} \mathrm{C}$ but cannot be completed before reaching $160{ }^{\circ} \mathrm{C}$. The remaining crystallizable melt then transforms into the LC-phase which rapidly converts to $\alpha$-crystals.

It was furthermore found that the temperatures of formation of $\beta$ '-and $\alpha$-crystals are strongly grade-dependent, as concluded from available data in the literature [5, 24] as well as analysis of purposely-synthesized PBN samples [11]. It might be deduced that crystallization in this cooling-rate-/temperature-range is largely governed by the molar mass/intrinsic viscosity of the different samples, and also by the different amount and type 
of cyclic oligomers and low weight molecules. Crystallization below $160{ }^{\circ} \mathrm{C}$, in contrast, seems rather independent on the molecular characteristics and on possible differences regarding impurities. This observation may tentatively be interpreted by different nucleation mechanisms, as it is known that heterogeneous crystal nucleation is often dominant at low supercooling, while homogeneous crystal nucleation prevails at high meltsupercooling [25-27].

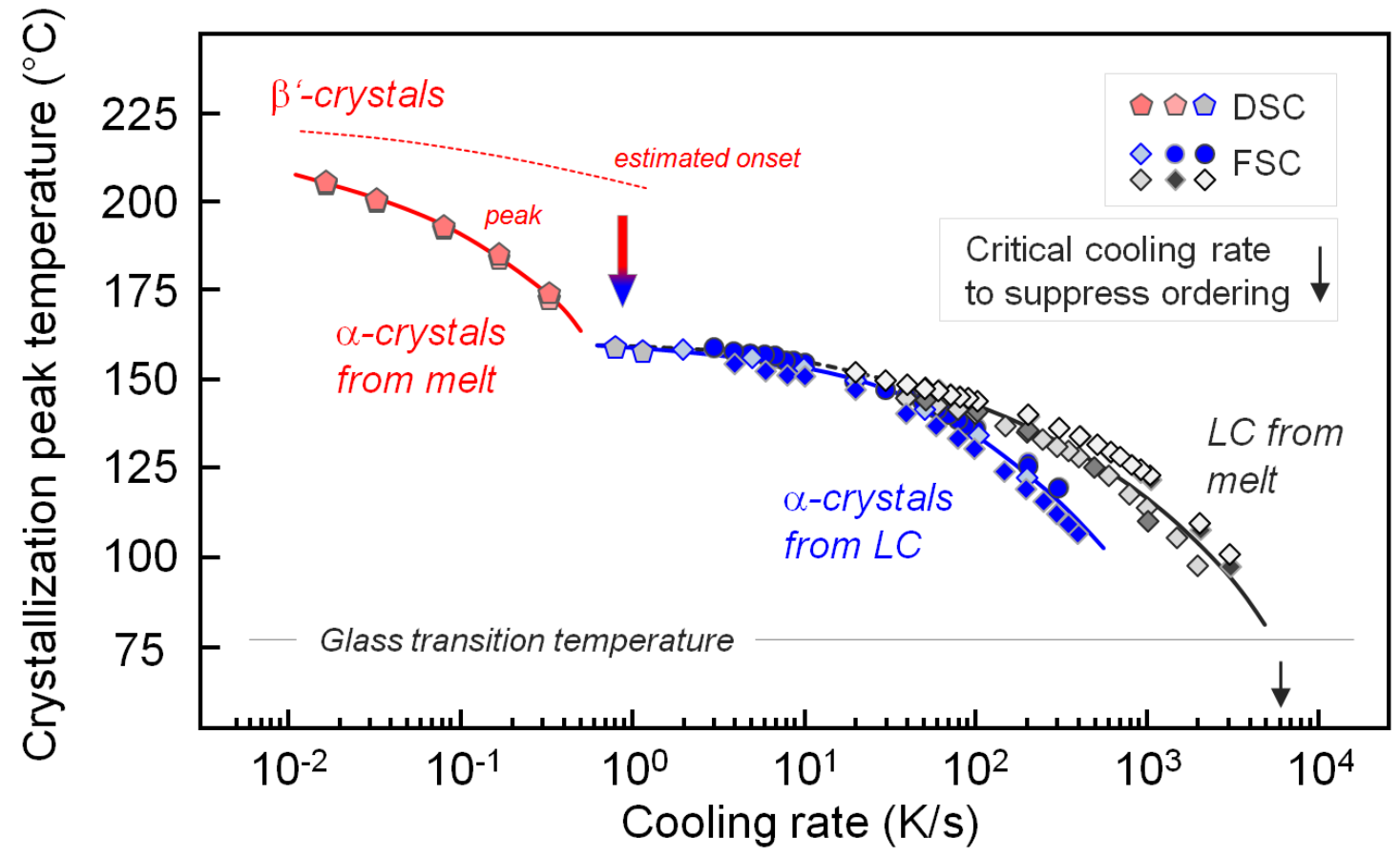

Figure 1: Crystallization peak temperature of PBN as a function of the cooling rate. Colorcoding is used to emphasize formation of $\beta$ '- and $\alpha$-crystals from the isotropic melt (red), of $\alpha$-crystals from the LC-phase (blue), and of the LC-phase (gray/black). The vertical black arrow indicates the critical cooling rate above which the isotropic melt vitrifies without prior formation of an ordered phase [3], and the horizontal line approximately represents the glass transition temperature [1]. Note that formation of $\beta$ '-crystals on very slow cooling, as indicated in the top left corner, was not experimentally proven in this work, but is well described in the literature [4-6].

If the cooling rate exceeds the (grade-specific) critical values which cause suppression of melt-crystallization at temperatures higher than about $160^{\circ} \mathrm{C}$, then mesophase develops and later transforms to $\alpha$-crystals, with LC-phase and $\alpha$-crystal formation denoted with black/gray and blue symbols and lines, respectively. With the dashed black line between 1 and $20 \mathrm{~K} / \mathrm{s}$ (drawn almost parallel to the blue line) is indicated that the LC-phase has not been detected in this cooling-rate range due to the extremely fast transformation into $\alpha$ - 
crystals; as such the assignment of the detected low-temperature exothermic event to the LC-to- $\alpha$-crystal transformation still requires evidence (blue symbols between 1 and 10 $\mathrm{K} / \mathrm{s}$ ). However, by inspection of the cooling-rate dependence of crystallization temperatures below and above about $1 \mathrm{~K} / \mathrm{s}$, it seems obvious that there is a change in the mechanism of $\alpha$-crystal formation, which becomes much faster when forming from the LC-mesophase (as evidenced by the less pronounced temperature-dependence of the ordering process: formation of $\alpha$-crystals is almost independent on the cooling rate between 1 and $10 \mathrm{~K} / \mathrm{s}$ ). Despite mesophase was not directly detected in this cooling-rate range, the data perfectly agree with results obtained at cooling rates higher than $50 \mathrm{~K} / \mathrm{s}$, for which the twofold crystallization process of the melt via the mesophase has been proven $[16]$.

If the cooling rate is higher than $200-500 \mathrm{~K} / \mathrm{s}$ then the LC-mesophase does not convert to crystals anymore, and freezes below $70-80{ }^{\circ} \mathrm{C}$ at the glass transition temperatures of both the amorphous phase and the LC-phase. For the latter a value of $65^{\circ} \mathrm{C}$ has been suggested while reports about $T_{\mathrm{g}}$ of the relaxed melt differ in a wide range between 41 and $82{ }^{\circ} \mathrm{C}$ [13]. Finally, if the cooling rate exceeds few thousands $\mathrm{K} / \mathrm{s}$ then even mesophase formation is suppressed, and the entire melt vitrifies without formation of any ordered phase. In Figure 1, the vertical black arrow indicates the critical cooling rate above which the isotropic melt vitrifies without prior formation of an ordered phase [3], and the gray horizontal line represents the glass transition temperature, though being just included in the plot as a rough estimate.

It is worth noting that the various routes of ordering on cooling the melt to below $T_{\mathrm{g}}$, namely (i) crystallization directly from the melt, (ii) crystallization via intermediate mesophase formation, and (iii) LC-phase formation without crystallization, overlap in certain cooling-rate ranges, which complicates the obtainment of quantitative information about critical cooling rates needed to enforce a specific crystallization path. For example, if high-temperature crystallization (red data points) cannot be completed since the melt is cooled too fast, then at temperatures lower than about $160{ }^{\circ} \mathrm{C}$ LC-phase formation occurs followed by a quick transformation to crystals as long as the cooling rate is lower than few hundred K/s (blue data points). Naturally, also the transition of the mesophase into $\alpha$ crystals is kinetically controlled, which is easily recognized by the decrease of the transformation temperature with increasing cooling rate. This implies that on increasing the cooling rate from about 50 to about $500 \mathrm{~K} / \mathrm{s}$ there is an increasing suppression of formation 
of $\alpha$-crystals such that at room temperature a mixture of amorphous phase, LC-mesophase and $\alpha$-crystals will be present.

Information about the morphology of PBN solidified using different thermal pathways was collected by POM. Figures 2-4 show POM micrographs focusing on imaging $\beta$ spherulites, $\alpha$-spherulites, and LC-mesophase, respectively, that is, the structures which develop at increasingly higher rate of cooling the melt according to Figure 1. Formation of $\beta$-spherulites has been enforced by isothermal annealing the melt at various temperatures between 200 and $220^{\circ} \mathrm{C}$. In the specific example of Figure 2, the sample was held at 215 ${ }^{\circ} \mathrm{C}$ for a period of $40 \mathrm{~min}$ which led to development of dendritically grown $\beta$-spherulites with a size of around $20 \mu \mathrm{m}$; the link between the specific spherulite structure and presence of $\beta$ '-crystals was evidenced by XRD as reported in the literature [6]. Further annealing would lead to a space-filled spherulitic morphology which, however, is not shown in favor of demonstrating the characteristic morphological differences between the LC-mesophase and $\alpha$-spherulites. After annealing the melt at $215^{\circ} \mathrm{C}$ for $40 \mathrm{~min}$, allowing $\beta$ '-spherulite formation, the remaining melt was either quenched to room temperature to observe the mesophase (left image), or transferred to $190{ }^{\circ} \mathrm{C}$ to permit growth of $\alpha$-structure (right image); the XRD pattern of a sample crystallized at about $190{ }^{\circ} \mathrm{C}$ on slow cooling at 0.1 $\mathrm{K} / \mathrm{s}$ (see Figure 1) is provided below and confirms the formation of $\alpha$-crystals at this temperature.
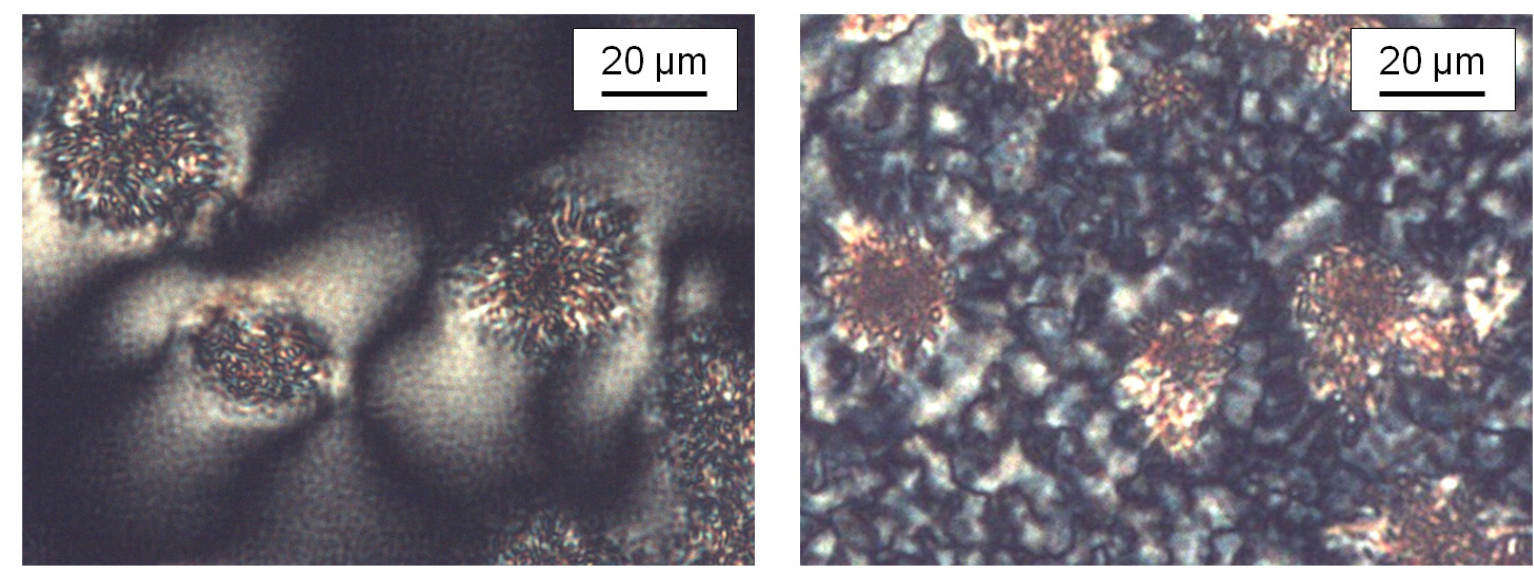

Figure 2: POM micrographs of samples of PBN solidified by rapid cooling the melt from $290{ }^{\circ} \mathrm{C}$ to $215^{\circ} \mathrm{C}$, followed by isothermal annealing for $40 \mathrm{~min}$ for incomplete growth of $\beta$-spherulites. The remaining melt was then either quenched to room temperature (left image), or quickly cooled to $190^{\circ} \mathrm{C}$, to continue crystallization for $10 \mathrm{~min}$ (right image). 
Figure 3 shows the semicrystalline morphology of PBN solidified by annealing at temperatures between 190 and $140{ }^{\circ} \mathrm{C}$ for a period of time which allows completion of the ordering process. Crystallization at temperatures between 190 and $160^{\circ} \mathrm{C}$ (top-row images) leads to the formation of spherulites, with the observed Maltese cross indicating well-organized radial alignment of lamellar crystals. The size of spherulites decreases with decreasing crystallization temperature as it is expected due to the increase of the nucleation rate [28]. According to the literature [4-6] it is expected that at temperatures below about $200{ }^{\circ} \mathrm{C}$, formation of $\beta$ '-crystals is negligible and quickly fading with decreasing crystallization temperature. As such, we assume that the spherulites shown in Figure 3 contain $\alpha$-crystals, which is also supported by a simple comparison with the dendritic spherulite morphology obtained on crystallization above $200{ }^{\circ} \mathrm{C}$ (see Figure 2).
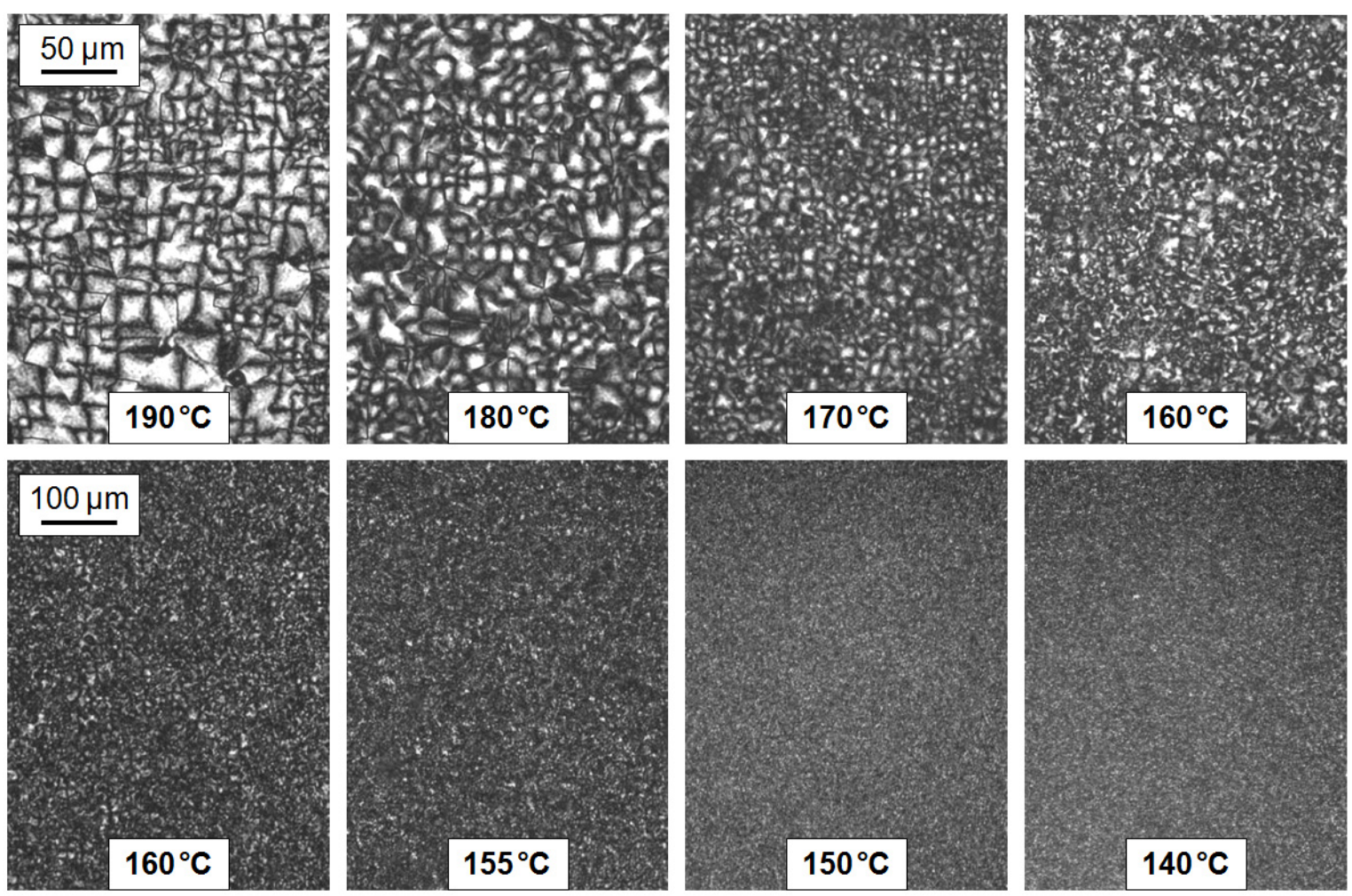

Figure 3: POM micrographs of samples of PBN solidified by rapid cooling the melt from $290{ }^{\circ} \mathrm{C}$ to temperatures between 190 and $140{ }^{\circ} \mathrm{C}$, followed by isothermal annealing. The annealing time was $20 \mathrm{~min}$ in case of crystallization at $190{ }^{\circ} \mathrm{C}$, otherwise $10 \mathrm{~min}$ at lower temperatures.

If the crystallization temperature is lower than $160{ }^{\circ} \mathrm{C}$, then individual spherulites cannot be detected anymore. Instead, a grainy structure is observed, pointing either to a distinctly increased concentration of heterogeneous nuclei, not allowing lateral growth of large superstructures/spherulites, or to a different crystallization pathway. The crystallization 10 
temperatures of Figure 1, along with the previous study [16], suggest that in this temperature range crystallization proceeds via the fast formation of the LC-mesophase. If we assume a homogeneous nucleation mechanism for the LC-phase, this could tentatively explain the $\alpha$-phase morphology formed at temperatures around $150{ }^{\circ} \mathrm{C}$. However, further investigation is needed to provide an ultimate explanation of the observation.

Finally, Figure 4 shows POM images obtained on PBN samples solidified during rapid cooling of the melt by quenching into ice-water. Though the temperature-time profile during cooling was not directly measured, the images prove that ordering was not completely suppressed indicating that the cooling rate certainly was lower than $6000 \mathrm{~K} / \mathrm{s}$ (see Figure 1, vertical arrow). In fact, in case of complete suppression of crystallization/ordering, absence of any macroscopic birefringence pattern would have been expected which obviously is not the case. In contrast, the images suggest the formation of the LC-mesophase since spherulites and grains, detected on solidification at temperatures higher than $140^{\circ} \mathrm{C}$, are absent. Referring to Figure 1, and earlier research about the kinetics of non-isothermal crystallization of PBN [16], we deduce that the cooling rate in this quenching experiment exceeded few hundred $\mathrm{K} / \mathrm{s}$, leading to mesophase formation and freezing of the system slightly below $80{ }^{\circ} \mathrm{C}$ without prior transformation of the LC-phase into $\alpha$-crystals. The images clearly demonstrate the formation of a Schlieren texture, which is typical for nematic LC-phases, but also occasionally reported for smectic LC-phases [29-32]; the Schlieren texture observed in this work is characterized by the presence of multiple disclination lines and point singularities, from which typically four brushes are originating.
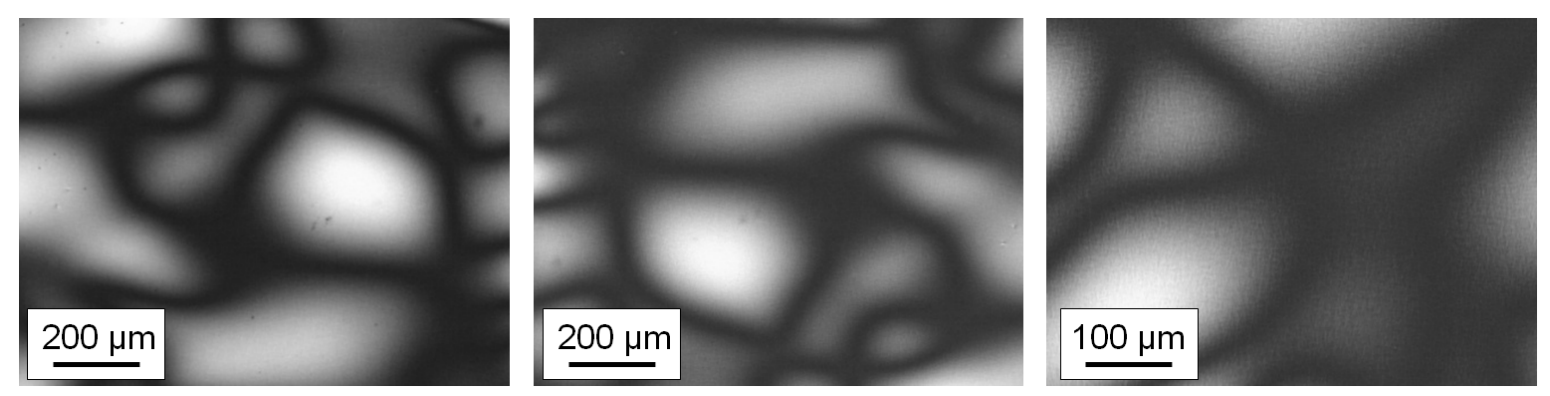

Figure 4: POM micrographs of samples of PBN solidified by rapid cooling the melt from $290{ }^{\circ} \mathrm{C}$ by quenching into ice-water.

In order to confirm that the observed Schlieren texture represents a smectic LC-phase, XRD has been employed, attempting to detect a smectic-periodicity peak. In advance to 
the prior pioneering work about the mesophase of PBN [13], in the present study the LCphase has been prepared in an FSC, which allows the application of a well-defined solidification path, beyond the ballistic cooling experiment using ice-water. In detail, a series of FSC samples with a thickness and lateral dimension of about 20 and $300 \mu \mathrm{m}$, respectively, has been prepared by cooling the melt at various rates between 0.1 and 1000 $\mathrm{K} / \mathrm{s}$, and then analyzed regarding the X-ray structure.

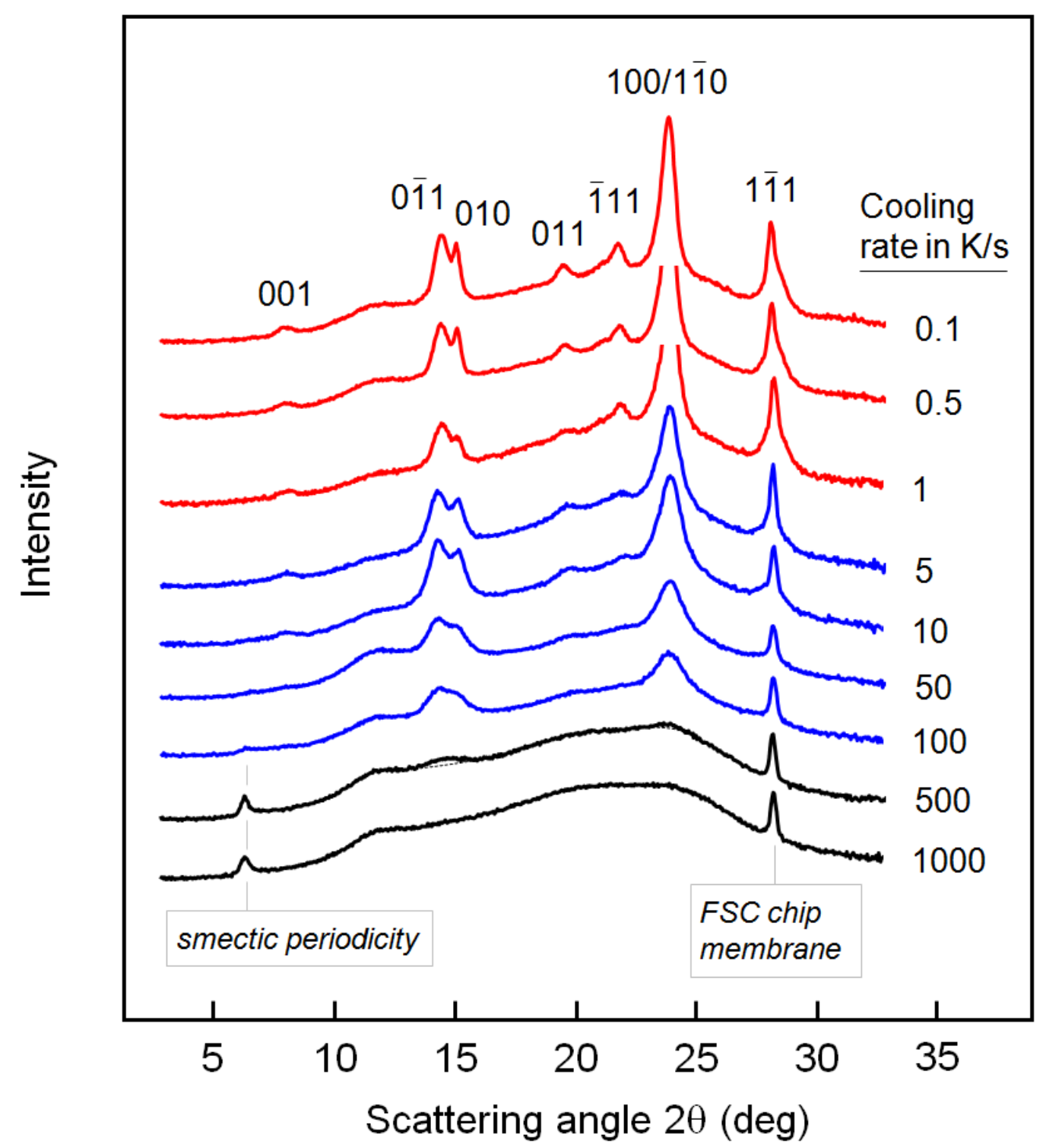

Figure 5: XRD curves obtained on samples of PBN prepared by solidification of the melt at the indicated cooling rates in an FSC. Diffraction peaks of the $\alpha$-phase are labeled according to $[6,13]$. 
Figure 5 shows XRD curves, color-coded in agreement with the data of Figure 1, emphasizing preferred formation of $\alpha$-crystals from the melt on slow cooling at rates of $0.1,0.5$, and $1 \mathrm{~K} / \mathrm{s}$ (red), formation of $\alpha$-crystals from the mesophase on cooling at rates between 5 and $100 \mathrm{~K} / \mathrm{s}$ (blue), and formation of mesophase on cooling at 500 and $1000 \mathrm{~K} / \mathrm{s}$ (black). Most important in the context of present work is the observation of the smecticperiodicity peak at a scattering angle slightly higher than 6 deg $2 \theta$ on cooling PBN faster than about $100 \mathrm{~K} / \mathrm{s}$. As such, the Schlieren texture observed by POM (see Figure 4) does not correspond to a nematic but rather to a smectic LC-phase. While in case of the sample cooled at $1000 \mathrm{~K} / \mathrm{s}$ peaks associated to $\alpha$-crystals are not detected, the XRD curve obtained on the sample cooled at $500 \mathrm{~K} / \mathrm{s}$ shows minor intensity increases at scattering angles around 15 and $24 \operatorname{deg} 2 \theta$, indicating that a small part of the mesophase converted to crystals (see dotted lines). Traces of the smectic-periodicity peak can be detected on cooling at rates down to $50 \mathrm{~K} / \mathrm{s}$. In other words, cooling between 50 and $500 \mathrm{~K} / \mathrm{s}$ leads to formation of a multi-phase structure consisting of amorphous phase, LC-phase, and $\alpha$ crystals. The latter phase is identified by the characteristic scattering peaks labeled at the top curve $[6,13]$.

For a further confirmation of the structural and morphological assignment, the POM micrographs of PBN samples crystallized in the FSC at rates of 1, 10, 100, and $1000 \mathrm{~K} / \mathrm{s}$ are provided in Figure 6. The obtained images confirm the morphological observations provided with Figures 3 and 4, and support, together with the XRD scans of Figure 5, the above provided interpretation of crystallization events in Figure 1. Cooling the material at a rate of $1 \mathrm{~K} / \mathrm{s}$ leads to spherulitic growth of $\alpha$-crystals directly from the melt, with the transition beginning above $200{ }^{\circ} \mathrm{C}$ (see dashed red line in Figure 1) and stretching down to $160{ }^{\circ} \mathrm{C}$ where a negligible fraction may have formed via the LC-mesophase. If the cooling rate is increased to $10 \mathrm{~K} / \mathrm{s}$, then a similar fine-grained structure is observed as was shown in the bottom image-row of Figure 3. The structure consists of amorphous phase and $\alpha$ crystals, similar as after cooling at $1 \mathrm{~K} / \mathrm{s}$, however, with the crystalline phase likely having being formed from the LC-mesophase. Cooling PBN at a rate of $100 \mathrm{~K} / \mathrm{s}$, according to the XRD curve of Figure 5, leads to a multiphase morphology containing amorphous phase, LC-mesophase, and $\alpha$-crystals. The corresponding POM image in Figure 6 shows both Schlieren/disclination lines originating from the mesophase and fine grains, which are assumed being indicative of $\alpha$-crystals. In other words, the LC-mesophase only partly transforms to $\alpha$-crystals. It has been posted above that with increasing cooling rate the 
transition of the mesophase into $\alpha$-crystals is increasingly suppressed, being completely absent on cooling at $1000 \mathrm{~K} / \mathrm{s}$ (see bottom XRD curve in Figure 5). In that case the FSC sample reveals a distinct Schlieren texture, similar to the one observed after quenching in ice-water as shown in Figure 4. Note that the spotty appearance of the FSC sample (bottom right image in Figure 6) is due to the structure of the sensor membrane, and not the sample itself.
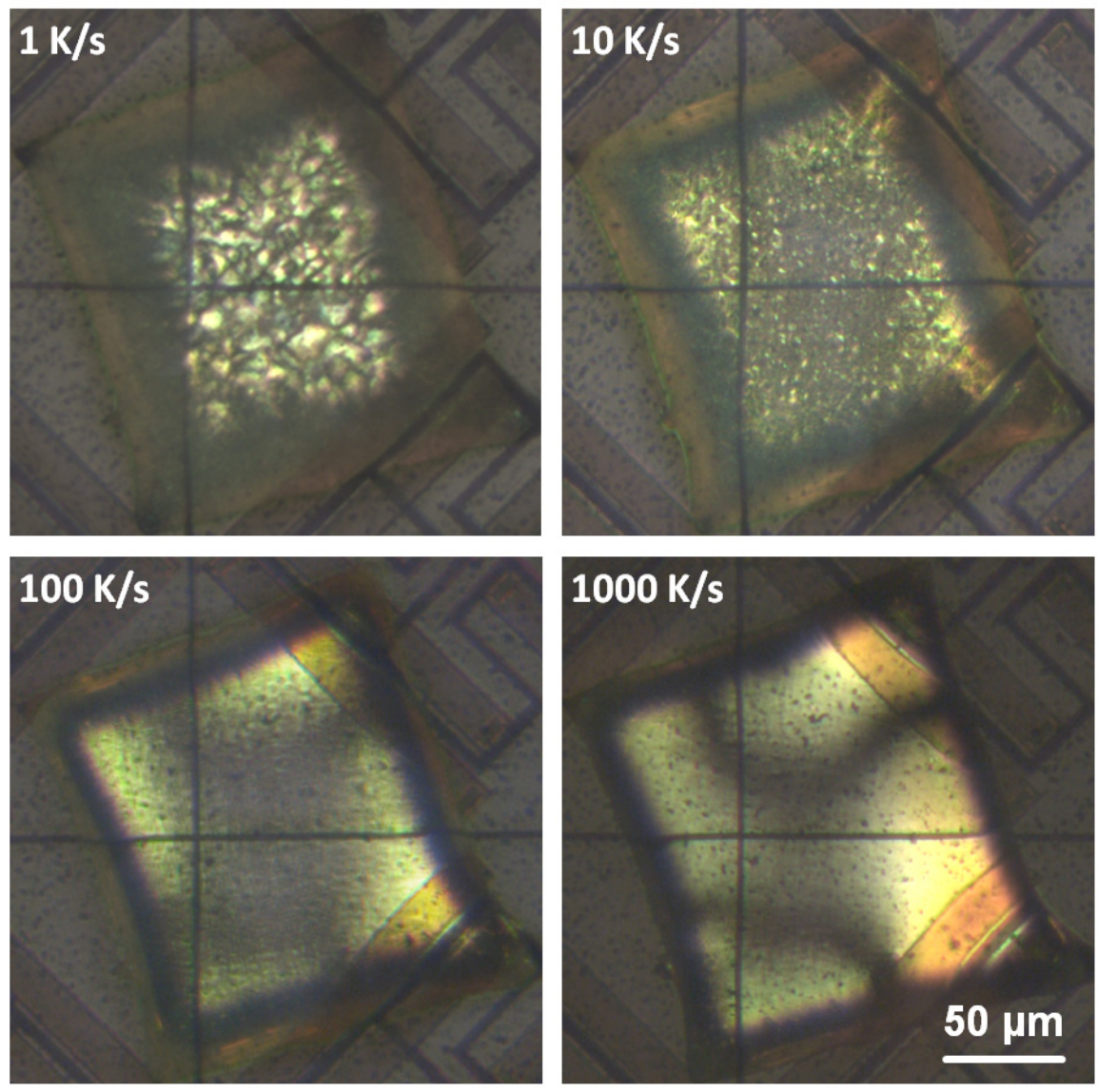

Figure 6: POM reflection-mode micrographs of samples of $\mathrm{PBN}$ solidified on cooling at rates of $1,10,100$, and $1000 \mathrm{~K} / \mathrm{s}$ in an FSC.

\section{Conclusions}

Poly(butylene naphthalate) is an engineering thermoplastic polymer which displays a distinct polymorphism, includes several crystalline structures and a liquid-crystalline mesophase. The resulting ordered structure upon cooling the melt critically depends on the thermal history. In this work, we investigated the supermolecular morphology of the various ordered phases, coupling fast scanning chip calorimetry (FSC) with ex-situ 
polarized-light optical microscopy (POM) and microfocus-beam X-ray diffraction (XRD). The explored range of cooling rates covers about four orders of magnitude, from $10^{-1}$ up to $10^{3} \mathrm{~K} / \mathrm{s}$.

At low cooling rates, the $\beta$ '- and $\alpha$ - crystalline phases crystallize directly from the melt, with the latter prevailing with increasing supercooling. Crystallization at rather low supercooling of the melt, at temperatures higher than about $200{ }^{\circ} \mathrm{C}$, leads to slow and irregular spherulitic growth of $\beta$ '-crystals with the spherulites not showing a distinct Maltese cross in POM. When the cooling rate is higher than about $1 \mathrm{~K} / \mathrm{s}$, then melt transforms first into a liquid-crystalline mesophase which subsequently turns into $\alpha$ crystals. This crystallization from an ordered mesomorphic state is kinetically controlled, and may occur only partially with increasing cooling rate and even is totally prevented if the melt is quenched at rates above about $500 \mathrm{~K} / \mathrm{s}$. In the latter situation, XRD shows the existence of a smectic liquid-crystalline glass at room temperature, possessing a characteristic Schlieren texture when observed by POM. The $\alpha$-crystals which formed via the mesophase lead to a grainy birefringent structure with features not distinguishable at the micrometer length scale. The two morphologies might coexist in the sample, when the transition to the ultimately stable crystalline state is partially suppressed.

We note that the structural and morphological features revealed in this work are relevant in the context of structure formation during industrial processing of $\mathrm{PBN}$, where cooling rates of tens and hundreds $\mathrm{K} / \mathrm{s}$ may be encountered. The coupling of chip calorimetry with XRD and POM characterization is essential for a deeper understanding of the exact crystallization pathway in complex situations as presented here for PBN. Moreover, the possibility of accessing high cooling rates by FSC allows the study of novel monotropic liquid crystalline polymers, such as PBN, where the mesophase can only form if the fast direct melt-crystallization is by-passed upon quenching.

\section{Acknowledgments}

One of the authors (QD) is thankful for financial support by the China Scholarship Council (CSC). The authors are grateful to Teijin Shoji Europe GmbH for supply of samples. 


\section{References}

[1] https://www.teijin.com/products/resin/products/pbn/

[2] http://www.yasojima.co.jp/en/zairyo/pbn/index.html; https://www.plasticstoday.com/content/pbn-resin-targets-diverseapplications/86636001217424; https://www.teijin.com/products/resin/products/pbn/

[3] K. Nishida, E. Zhuravlev, B. Yang, C. Schick, Y. Shiraishi, Vitrification and crystallization of poly(butylene-2,6-naphthalate), Thermochim. Acta 603 (2015) $110-115$.

[4] T. Chiba, S. Asai, W. Xu, M. Sumita, Analysis of crystallization behavior and crystal modifications of poly(butylene-2,6-naphthalene dicarboxylate), J. Polym. Sci, Polym. Phys. 37 (1999) 561-574.

[5] M.-Y. Ju, F.-C. Chang, Multiple melting behavior of poly(butylene-2,6-naphthalate), Polymer 42 (2001) 5037-5045.

[6] M.-Y. Ju, J.- M. Huang, F.-C. Chang, Crystal polymorphism of poly(butylene-2,6naphthalate) prepared by thermal treatments, Polymer 43 (2002) 2065-2074.

[7] G. Z. Papageorgiou, V. Tsanaktsis, D. N. Bikiaris, Crystallization of poly(butylene2,6-naphthalate-co-butylene adipate) copolymers: regulating crystal modification of the polymorphic parent homopolymers and biodegradation, Cryst. Eng. Comm. 16 (2014) 7963-7978.

[8] H. Watanabe, Stretching and structure of polybutylene-naphthalene-2, 6dicarboxylate films, Kobunshi Ronbunshu 33 (1976) 229-336.

[9] T. Yamanobe, H. Matsuda, K. Imai, A. Hirata, S. Mori, T. Komoto, Structure and physical properties of naphthalene containing polyesters I. Structure of poly(butylene 2,6-naphthalate) and poly(ethylene 2,6-naphthalate) as studied by solid state NMR spectroscopy, Polymer J. 28 (1996) 177-183.

[10] H. Koyano, Y, Yamamoto, Y. Saito, T. Yamanobe, T. Komoto, Crystal structure of poly(butylene-2,6-naphthalate), Polymer 39 (1998) 4385-4391.

[11] M. Soccio, N. Lotti, L. Finelli, A. Munari, Equilibrium melting temperature and crystallization kinetics of $\alpha$ - and $\beta^{\prime}$-PBN crystal forms, Polymer J. 44 (2012) 174180.

[12] S.C. Lee K.H. Yoon, J.H. Kim, Crystallization kinetics of poly(butylene 2,6naphthalate) and its copolyesters, Polymer 29 (1997) 1-6.

[13] T. Konishi, K. Nishida, G. Matsuba, T. Kanaya, Mesomorphic phase of poly(butylene-2,6-naphthalate), Macromolecules 41 (2008) 3157-3161.

[14] M. Tokita, J. Watanabe, Several interesting fields exploited through understanding of polymeric effects on liquid crystals of main-chain polyesters, Polymer J. 38 (2006) 611-638. 
[15] B. Wunderlich, J. Grebowicz, Thermotropic mesophases and mesophase transitions of linear, flexible macromolecules, Adv. Polym. Sci. 60/61(1984) 1-59.

[16] D. Cavallo, D. Mileva, G. Portale, L. Zhang, L. Balzano, G.C. Alfonso, R. Androsch, Mesophase-mediated crystallization of poly(butylene-2,6-naphthalate): an example of Ostwald's rule of stages, ACS Macro Letters 1 (2012) 1051-1055.

[17] W. Ostwald, Studien über die Bildung und Umwandlung fester Körper, Z. Phys. Chem. 22 (1897) 286-330.

[18] G. Smyth, E.M. Valles, S.K. Pollack, J. Grebowicz, P.J. Stenhouse, S.L. Hsu, W.J. MacKnight, Development of crystallinity in a polyurethane containing mesogenic units. 1. Morphology and mechanism, Macromolecules 23 (1990) 3389-3398.

[19] A.J. Jing, O. Taikum, C.Y. Li, F.W. Harris, S.Z.D. Cheng, Phase identifications and monotropic transition behaviors in a thermotropic main-chain liquid crystalline polyether, Polymer 43 (2002) 3431-3440.

[20] D. Heberer, A. Keller, V. Percec, Interrelation between crystallization and liquid crystal formation: a calorimetric and polarizing microscopical study on a monotropic polymer system, J. Polym. Sci., Polym. Phys. 33 (1995) 1877-1894.

[21] S.O. Kim, C.M. Koo, I.J. Chung, H.-T. Jung, Phase behavior, crystallization kinetics, and morphology of monotropic liquid crystalline poly (ester-imide)s with a decamethylene spacer, Macromolecules 34 (2001) 8961-8967.

[22] Y.Y. Cheng, P. Cebe, M. Capel, H. Schreuder-Gibson, A. Bluhm, W. Yeomans, Small-angle X-ray scattering study of liquid crystalline polycarbonates based on $\alpha$-methyl stilbene mesogen and methylene-containing flexible spacer, J. Polym. Sci., Polym. Phys. 33 (1995) 2331-2341.

[23] R. Pardey, S.S. Wu, J. Chen, F.W. Harris, S.Z.D. Cheng, A. Keller, J. Aducci, J.V. Facinelli, R.W. Lenz, Liquid crystal transition and crystallization kinetics in poly(ester imide)s, Macromolecules 27 (1994) 5794-5802.

[24] G. Papageorgiou, G. Karayannidis, D. Bikiaris, A. Stergiou, G. Litsardakis, S. Makridis, Wide-angle x-ray diffraction and differential scanning calorimetry study of crystallization of poly(ethylene naphthalate), poly(butylene naphthalate), and their copolymers, J. Polym. Sci., Polym. Phys. 42 (2004) 843-860.

[25] A. Toda, R. Androsch, C. Schick, Insights into polymer crystallization and melting by fast scanning chip calorimetry, Polymer 91 (2016) 239-263.

[26] R. Androsch, C. Schick, Crystal nucleation of polymers at high supercooling of the melt, Adv. Polym. Sci. 276 (2017) 257-288.

[27] C. Schick, R. Androsch, J.W.P. Schmelzer, Homogeneous crystal nucleation in polymers, J. Phys.: Condensed Matter 29 (2017) 453002 (35 pp) (2017).

[28] B. Wunderlich, Macromolecular Physics, Vol. 2, Crystal nucleation, growth, annealing, New York, Academic Press, 1976. 
[29] P.G. de Gennes, J. Prost, The Physics of Liquid Crystals, Oxford University Press, New York 1993.

[30] Sackmann, D. Demus, The Polymorphism of Liquid Crystals, Mol. Cryst. 2 (1966) 81-102.

[31] J. Nehring, A. Saupe, On the schlieren texture in nematic and smectic liquid crystals, J. Chem. Soc., Faraday Trans. 2: Mol. Chem. Phys. 68 (1972) 1-15.

[32] D. Demus, Schlieren textures in smectic liquid crystals, Kristall und Technik 10 (1975) 933-946. 